Document downloaded from:

http://hdl.handle.net/10251/150057

This paper must be cited as:

Blandez, JF.; Santiago-Portillo, A.; Navalón Oltra, S.; Gimenez Marques, M.; Alvaro Rodríguez, MM.; Horcajada, P.; García Gómez, H. (2016). Influence of functionalization of terephthalate linker on the catalytic activity of UiO-66 for epoxide ring opening. Journal of Molecular Catalysis A Chemical. 425:332-339. https://doi.org/10.1016/j.molcata.2016.10.022

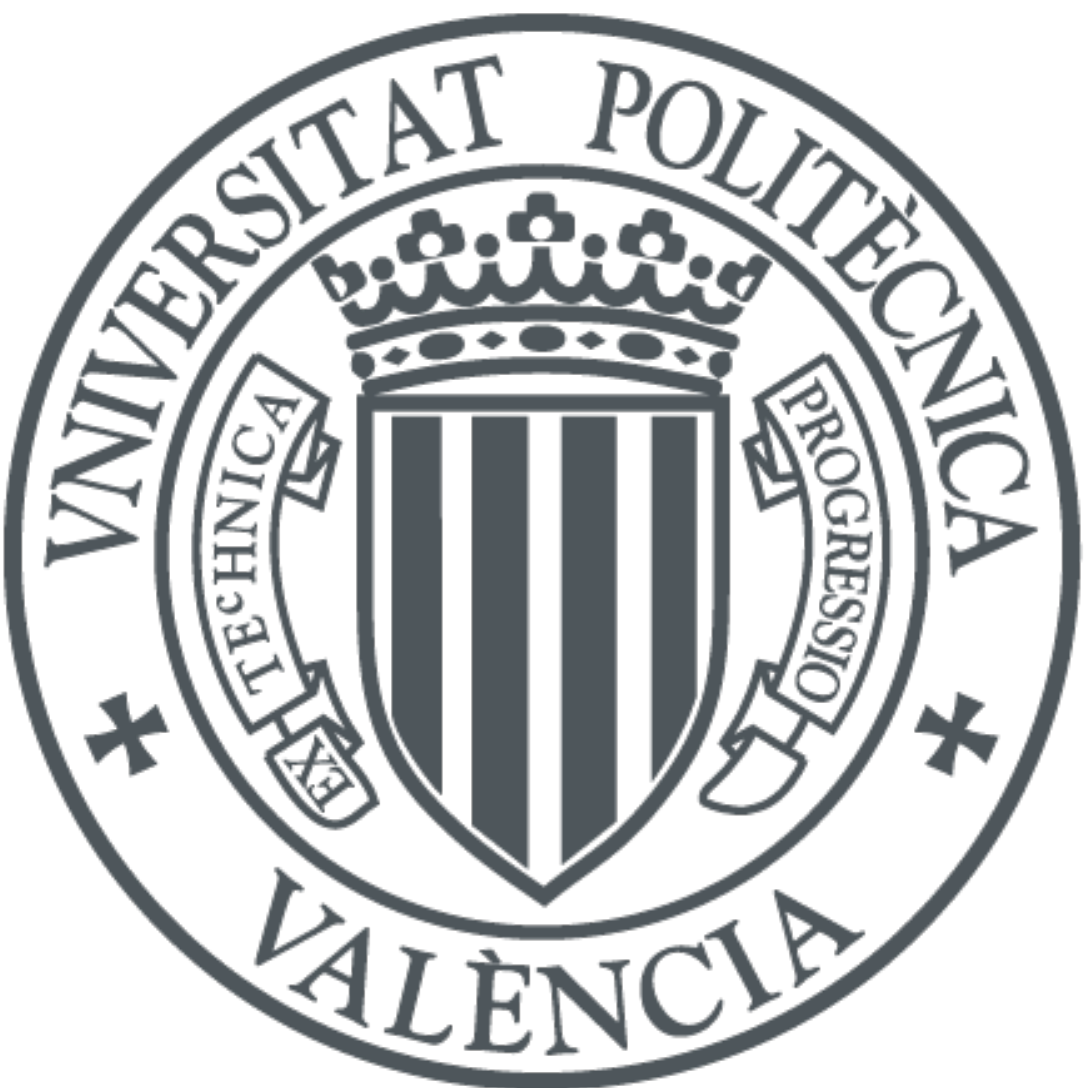

The final publication is available at

https://doi.org/10.1016/j.molcata.2016.10.022

Copyright Elsevier

Additional Information 


\title{
Influence of the functionalization of terephthalate linker on the catalytic activity of UiO-66 for epoxide ring opening.
}

\author{
Juan F. Blandez, ${ }^{[\mathrm{a}]}$ Andrea Santiago-Portillo, ${ }^{\mathrm{a}]}$ Sergio Navalón, ${ }^{[\mathrm{a}]}$ Mónica Giménez- \\ Marqués, ${ }^{[\mathrm{a}]}$ Mercedes Álvaro, ${ }^{[\mathrm{a}]}$ Patricia Horcajada, ${ }^{[\mathrm{c}, \mathrm{d}]}$ and Hermenegildo García*[a,b] \\ ${ }^{a} J . F$. Blandez, A. Santiago-Portillo, S.Navalón, M.Álvaro, H.García. Department of Chemistry and Instituto de Tecnología Química \\ (ITQ, CSIC-UPV) Universidad Politécnica de Valencia C/Camino de Vera, s/n, 46022, Valencia (Spain) \\ E-mail: hgarcia@qim.upv.es \\ ${ }^{b}$ Center of Excellence for Advanced Materials Research, King Abdulaziz University, Jeddah, Saudi Arabia. \\ ' Institut Lavoisier, UMR CNRS 8180 \\ Université de Versailles Saint-Quentin-en-Yvelines 45 avenue des Etats-Unis, 78035 Versailles cedex, France. \\ ${ }^{d}$ IMDEA Energy Avda. Ramon de la Sagra, 3, 28035 Mostoles, Madrid, Spain.
}

Keywords: heterogeneous catalysis, metal organic frameworks, UiO-66, epoxide ring opening.

Highlights:

\begin{abstract}
A series of five isostructural zirconium terephthalate UiO-66 metal organic frameworks bearing different functional groups on the terephthalate linker (UiO-66-X; $\mathrm{X}=\mathrm{H}, \mathrm{NH}_{2}, \mathrm{NO}_{2}, \mathrm{Br}, \mathrm{Cl}$,) have been successfully prepared and characterized. UiO-66-X materials were evaluated as heterogeneous catalysts for the epoxide ring opening of styrene oxide by methanol, observing an increase in the initial reaction rate from UiO-66-H to UiO$66-\mathrm{Br}$, over one order of magnitude. The reactivity order, however, does not follow a linear relationship between the Hammett constant value of the substituent and the initial reaction rate. UiO-66-Br exhibits a wide scope, its activity depending on the structure of epoxide and nucleophile. The absence of Zr leaching to the solution together with the preservation of the UiO-66-X crystallinity confirms the stability of the framework under the reaction conditions. Nevertheless, UiO-66 undergoes a progressive deactivation upon reuse that was attributed to a strong adsorption of the reaction product.
\end{abstract}

\section{Introduction}

Metal organic frameworks (MOFs), where the lattice is constituted by metal nodes coordinated to polycomplexant organic linkers [1],[2], are among the most porous materials, ranking at the top of the list of solids with high specific surface area [2],[3],[4]. The large metal content together with the fact that, in some MOFs, there are exchangeable coordination position around the metal nodes, make possible the use of these materials as heterogeneous catalysts for liquid phase reactions under conditions compatible with their chemical and structural stability [3],[5],[6],[7]. Particularly interesting as catalysts are the highly chemically and thermally stable metal carboxylates MIL-100, [8],[9] MIL-101[8],[10] and UiO-66 (UiO for University of Oslo) [11],[12] structures, among others [3],[4],[5],[6]. One of the major advantages of MOFs as catalysts is their chemical versatility, enabling to obtain isostructural materials based on diverse cations or organic linkers substituted by different functional groups [13]. In particular, the electron donating or withdrawing ability of the functional groups on the aromatic linkers can modulate the electron density of the complexing groups (e.g. carboxylates) coordinated to the cations and, consequently, influence the catalytic activity of the MOF through controlling the electron density of the active metal site [12],[14],[15]. As previously shown, the ligand functionalization of the microporous UiO-66, a cubic close packed structure based on $\mathrm{Zr}_{6} \mathrm{O}_{4}(\mathrm{OH})_{4}$ oxoclusters linked together by terephthalate linkers, strongly affects its Lewis acid catalytic activity [15]. Attention has to be paid, because the influence of the substituent probably overlaps with other possible differences in crystallinity and differences in the density of lattice defects [16]. Considering the large number of appealing liquid phase reactions that can be promoted by MOF-based solid catalysts, it is of high interest to expand this type of studies to other class of industrially relevant reactions [4],[6],[7],[17]. Epoxide ring opening, particularly by $\mathrm{H}_{2} \mathrm{O}$ as nucleophile, is one of the industrially more important reactions, since epoxides of simple alkenes are produced in very large amounts. Also from the synthetic point of view, epoxide opening is a general reaction of obtain vic-diols, $\alpha$-aminoalcohols, halohydrins and even for C-C bond formation.

In the present manuscript, we have prepared and characterized a series of five isostructural UiO-66 solids based on functionalized terephthalate ligands, exhibiting contrasting electron withdrawing or donating effects, for the evaluation of their catalytic activity in the epoxide ring opening by alcohols and amines. Although there 
are many homogeneous as well heterogeneous Lewis acid catalysts for this reaction, whose mechanism either $\mathrm{S}_{\mathrm{N}} 1$ or $\mathrm{S}_{\mathrm{N}} 2$, has been well studied, the purpose of the present work is to show how the presence of substituents on the terephthalic acid linkers modifies the catalytic activity [18]. Therefore the goal of the present study is to illustrate how the presence of substituents can modulate the activity of MOFs as solid catalyst for the particular case of epoxide ring opening.

\section{Experimental}

\subsection{Materials}

All the reagents and solvents used in this work were of analytical or HPLC grade and supplied by SigmaAldrich.

\subsection{Catalyst preparation.}

The series of UiO-66-X solids were prepared following reported procedures [19]. Briefly, the corresponding terephthalic acid derivative $(1.0 \mathrm{mmol})$ and $\mathrm{ZrCl}_{4}(0.233 \mathrm{~g}, 1 \mathrm{mmol})$ were added to a Teflon-lined autoclave containing $3 \mathrm{~mL}$ of dimethylformamide (DMF). This autoclave was heated at the temperature and time shown in Table 1. After cooling to room temperature, the resulting precipitate was washed with DMF and the solid was introduced in a Soxhlet system using methanol as solvent. Finally the solid was dried in an oven at $100{ }^{\circ} \mathrm{C}$ for $24 \mathrm{~h}$.

Table 1. Temperature and time employed in the synthesis of the various UiO-66-X solids [19].

\begin{tabular}{ccc}
\hline UiO-66-X & Temperature $\left({ }^{\circ} \mathrm{C}\right)$ & Time (h) \\
\hline UiO-66-H & 220 & 12 \\
UiO-66- $\mathrm{NO}_{2}$ & 220 & 24 \\
UiO-66- $\mathrm{NH}_{2}$ & 100 & 24 \\
UiO-66-Br & 100 & 20 \\
UiO-66-Cl & 100 & 24 \\
\hline
\end{tabular}

\subsection{Catalyst characterization.}

Fourier transformed infrared (FTIR) spectra were collected using a Nicolet 6700 instrument (Thermo scientific, USA) within the $4000-400 \mathrm{~cm}^{-1}$ range. Thermogravimetric analyses (TGA) were analyzed on a Perkin Elmer Diamond TGA/DTA STA 6000 in the $25-600{ }^{\circ} \mathrm{C}$ temperature range under a $5{ }^{\circ} \mathrm{C} \cdot \mathrm{min}^{-1}$ scan rate and an $\mathrm{O}_{2}$ flow of $20 \mathrm{~mL} \cdot \mathrm{min}^{-1}$. Powder X-ray diffraction (PXRD) patterns were collected in a D8 Advance Bruker diffractometer using $\mathrm{Cu} \mathrm{K \alpha 1}$ radiation $(\lambda=1.54056 \AA)$. Diffraction patterns were generally collected in the $3^{\circ}<2 \theta<30^{\circ}$ range with a typical step size of $0.02^{\circ}$ in continuous mode. $\mathrm{N}_{2}$ sorption isotherms were obtained at $77 \mathrm{~K}$ using a BELsorp Mini (Bel, Japan). Previous to the analysis, ca. $30 \mathrm{mg}$ of the sample were activated at $140{ }^{\circ} \mathrm{C}$ under primary vacuum for $3 \mathrm{~h}$.

\subsection{Catalytic experiments.}

Typically, the required amount of catalyst $(0.037 \mathrm{mmol}$ of $\mathrm{Zr})$ was added to a round-bottom flask $(25 \mathrm{~mL})$. To remove absorbed water in the catalysts, the MOFs were pre-treated by heating them at $150{ }^{\circ} \mathrm{C}$ under vacuum overnight. The required amount of alcohol was introduced $(2.5 \mathrm{~mL})$ into the flask and the system sonicated for $15 \mathrm{~min}$. After this time, the epoxide was added $(1 \mathrm{mmol})$. Subsequently, the reaction mixture was placed in a preheated bath at the required temperature (i.e. $120{ }^{\circ} \mathrm{C}$ ) and stirred magnetically. In the case of amine after the activation of the catalyst, acetonitrile $(2.5 \mathrm{~mL})$ was added as solvent, the catalyst was sonicated and finally, the amine $(1.0 \mathrm{mmol})$ and the styrene oxide $(1.0 \mathrm{mmol})$ were added. At the end of the reaction the catalyst was exhaustively washed with methanol to recover most of the adsorbed product. Product isolation was carried out by purification with flash column chromatography on silica gel using hexane: dichloromethane as eluent. The course of the reaction was periodically follow by extracting aliquot of the reaction mixture with a syringe, diluting in methanol and injecting the mixture immediately in GC (6890 Network GC system Agilent technologies) and using a calibration plot to determine the product concentration. 
Reuse experiments were performed under the above reaction conditions. At the end of the reaction, the catalyst was filtered ( $0.2 \mathrm{~nm}$ Nylon filter), the solid was introduced in a Soxhlet system and extracted using methanol as solvent for $24 \mathrm{~h}$. Then, the catalyst was dried at $100{ }^{\circ} \mathrm{C}$ for $24 \mathrm{~h}$. The used catalyst was also activated before subsequent use, as commented before.

\subsection{Product analysis.}

Previously filtered reaction aliquots were diluted in toluene. Subsequently, the aliquots were analyzed by gas chromatography using a flame ionization detector (GC-FID). Quantification was carried out by using calibration curves of authentic samples. In some cases selectivity was calculated using ${ }^{1} \mathrm{H}-\mathrm{NMR}$ spectra (Bruker $400 \mathrm{MHz}$ NMR spectrometer).

\subsection{Leaching experiments.}

At the end of the reaction, the catalyst was removed by filtration. The organic phase mixed with aqueous solution of $3 \mathrm{M} \mathrm{HNO}_{3}$, and the system heated at $80{ }^{\circ} \mathrm{C}$ for $24 \mathrm{~h}$. The presence of zirconium in the aqueous phase was analyzed by chemical analysis using an ICP-AES instrument.

\section{Results and discussion}

In order to evaluate the potential influence of terephthalate functionalization of UiO-66 on their catalytic activity, a series of five isostructural UiO-66-X solids bearing different functional groups on the aromatic ring of the terephthalate ligand ( $\mathrm{X}=\mathrm{H}, \mathrm{NH}_{2}, \mathrm{NO}_{2}, \mathrm{Br}, \mathrm{Cl}$ ) were solvothermally synthesized from zirconium(IV) chloride and the corresponding functionalized terephthalic acid in DMF, following the procedure reported in the literature [19]. The resulting UiO-66-X solids were characterized by PXRD, infrared spectroscopy (IR), TGA (see Figure S.1 in supporting information) and nitrogen adsorption measurements. All these data are in good agreement with the reported characterizations [19]. As expected, the Brunauer-Emmett-Teller (BET) surface area and pore volume of the bared UiO-66-H $\left(1150 \mathrm{~m}^{2} \cdot \mathrm{g}^{-1}\right.$ and $0.43 \mathrm{~cm}^{3} \cdot \mathrm{g}^{-1}$, respectively) decrease due to the presence of substituents. Table 2 lists the UiO-66-X solids prepared in the present study and their main compositional and textural properties. Crystal structure of the functionalized derivatives was confirmed by PXRD (Figure 1). All the PXRD patterns show the characteristic diffraction peaks of UiO-66 structure with, however, a different broadening of the Bragg reflections as a consequence of a different particle size and crystallinity. Similarly, the purity of the samples was confirmed by TGA, elemental analysis and IR (Table 2 and Figure S.2 in the supporting information).

Table 2. Analytic and textural data of UiO-66-X materials

\begin{tabular}{lccc}
\hline UiO-66-X & $\begin{array}{l}\text { BET surface area } \\
\left(\mathbf{m}^{\mathbf{2}} \cdot \mathbf{g}^{-1} \mathbf{)}\right.\end{array}$ & $\begin{array}{l}\text { Pore volume } \\
\left(\mathbf{c m}^{\mathbf{3}} \cdot \mathbf{g}^{-1} \mathbf{)}\right.\end{array}$ & Theoretical / Analized metal content (\%) \\
\hline $\mathrm{UiO}-66-\mathrm{H}$ & 1150 & 0.43 & $32.8 / 34.8$ \\
$\mathrm{UiO}-66-\mathrm{NO}_{2}$ & 750 & 0.30 & $28.2 / 34.8$ \\
$\mathrm{UiO}-66-\mathrm{NH}_{2}$ & 910 & 0.39 & $31.2 / 33.0$ \\
$\mathrm{UiO}-66-\mathrm{Br}$ & 745 & 0.46 & $25.6 / 28.7$ \\
$\mathrm{UiO}-66-\mathrm{Cl}$ & 610 & 0.23 & $29.2 / 35.8$
\end{tabular}


Then, the effect of ligand modification of UiO-66-X was evaluated on their catalytic activity for the epoxide ring opening by methanol. Finally the best catalyst UiO-66-Br was evaluated for the epoxide ring opening with alcohols and amines. A preliminary screening was initially carried out using styrene oxide and methanol as substrate and nucleophile, respectively. After reaction, the only product observed in all cases was 2-methoxy-2-phenylethanol, that corresponds to the product formed by $\mathrm{S}_{\mathrm{N}} 1$ mechanism [20]. In this type of mechanism, the epoxide oxygen should be activated by a Brønsted or Lewis acid site to generate a partial positive charge at the benzylic carbon, where the nucleophile methanol could attack. According with this simplistic mechanism, based on the observation of full selectivity towards a single product regioisomer, the key step of the catalysis might be the activation of styrene oxide by $\mathrm{Zr}_{6}(\mathrm{OH})_{4} \mathrm{O}_{4}$ oxoclusters, acting here as Lewis acids. Consequently, one could rationally expect that electron withdrawing substituents increase the acidity of the Zr nodes.
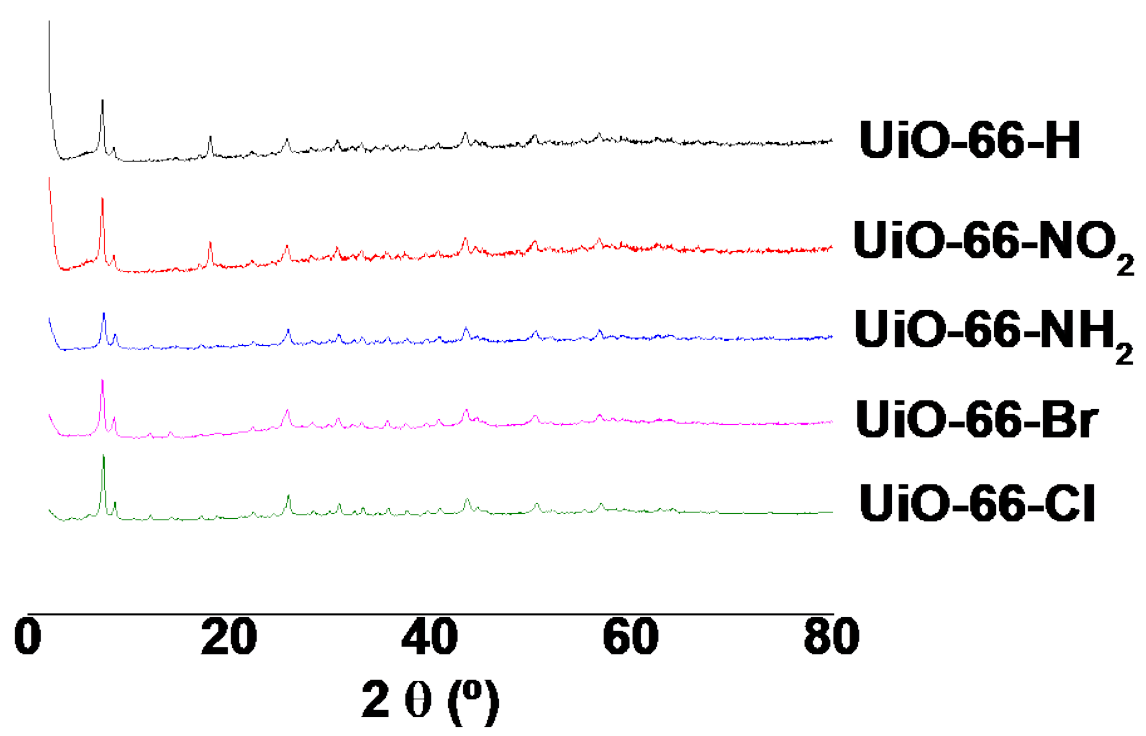

Figure 1. The figure shows the PXRD for the serie of five catalysts.

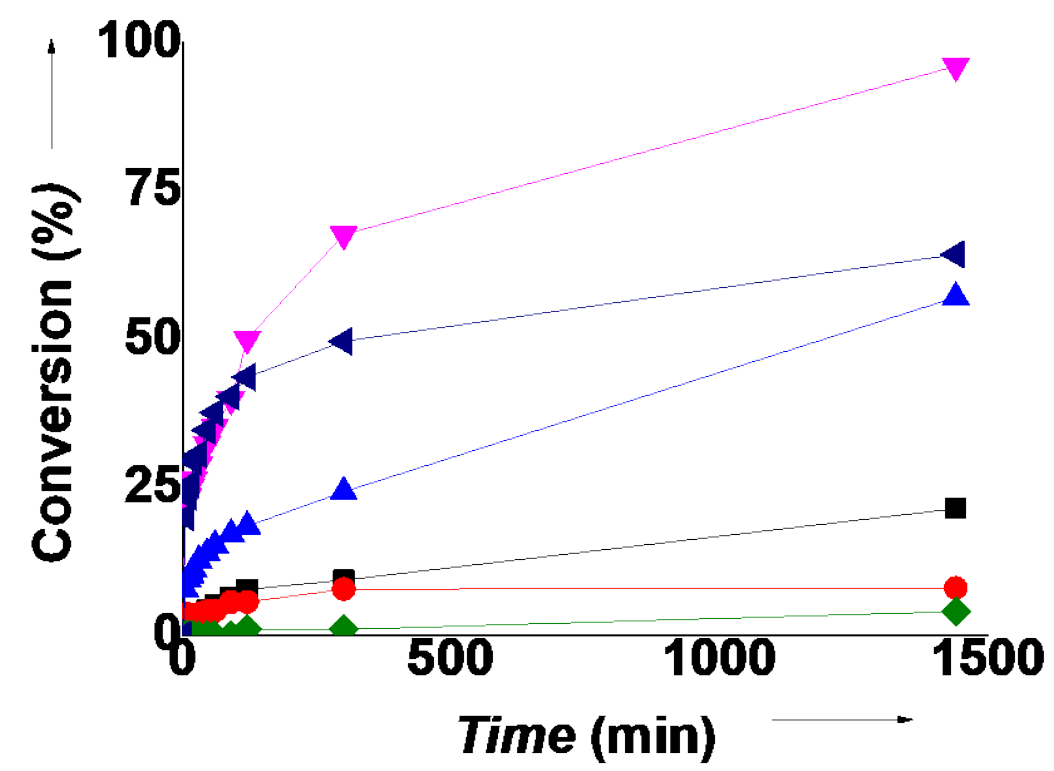

Figure 2. Time-conversion plots for the epoxide ring opening catalysed by a series of UiO-66-X.

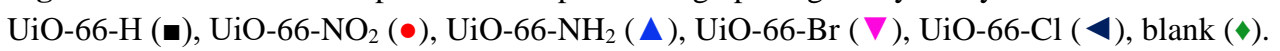
Reaction conditions: styrene oxide $(1 \mathrm{mmol})$, methanol $(5 \mathrm{~mL})$, internal standard $(0.5 \mathrm{mmol})$ and catalyst $(0.037 \mathrm{mmol}$ of metal) 
The time-conversion plots of styrene oxide disappearance for the series of UiO-66 are presented in Figure 2. Remarkably, catalytic performances exhibit important variations, depending on the functional group. Control experiments in absence of catalyst show that styrene oxide conversion under the used reaction conditions was below $5 \%$. In contrast, in presence of UiO-66-X solids, styrene oxide conversions were substantially higher and dependent on the UiO-66 functionalization, following the order of reactivity $\mathrm{Br}>\mathrm{Cl}>\mathrm{NH}_{2}>\mathrm{H}>\mathrm{NO}_{2}$. The initial reaction rates, determined from the slope of the time-conversion plots at zero time, are listed in Table 3.

Table 3. Initial reaction rate for the epoxide ring opening catalysed by the series of UiO-66- $\mathrm{X}^{[\mathrm{a}]}$

\begin{tabular}{cc}
\hline UiO-66-X & Initial rate $\left(\mathbf{m o l} \cdot \mathbf{s}^{\mathbf{1}}\right) \times \mathbf{1 0}^{\mathbf{6}}$ \\
\hline UiO-66-H & 9.6 \\
UiO-66- $-\mathrm{NO}_{2}$ & 20.0 \\
UiO-66-NH $\mathrm{NH}_{2}$ & 50.1 \\
UiO-66-Br & 143.1 \\
UiO-66-Cl & 128.8 \\
\hline
\end{tabular}

a) Reaction conditions: styrene oxide $(1 \mathrm{mmol})$, methanol $(5 \mathrm{~mL})$, internal standard $(0.5 \mathrm{mmol})$, catalyst (0.037 mmol of metal)

For a deeper understanding of the catalytic activity of the UiO-66-X derivatives, we further investigate the effect of the functionalized ligand on the electronic density of $\mathrm{Zr}_{6}(\mathrm{OH})_{4} \mathrm{O}_{4}$ clusters and, so, on its catalytic activity. If one could expect a direct correlation of the catalytic activity of UiO-66-X and the Hammett constant $\sigma$ or $\sigma+$ of the functional group of $\mathrm{UiO}-66-\mathrm{X}$, being roughly true for $\mathrm{Br}, \mathrm{Cl}$ and $\mathrm{H}$ derivatives, this is not the case for UiO-66- $\mathrm{NO}_{2}$ and UiO-66- $\mathrm{NH}_{2}$, which unexpectedly showed a much lower and higher activity, respectively, than anticipated. However, taking into account the general mechanism of nucleophilic attack on epoxides, we have to consider not only the inductive effect of the functional group on the Lewis acid sites, activating the styrene oxide (Scheme 1), but also: i) the presence of basic sites, potentially activating the nucleophile methanol, ii) the effect of steric hindrance coming from the volume of the substituent (effective steric effect, $v_{\text {ef: }}\left(\mathrm{H}<\mathrm{NH}_{2}<\mathrm{Cl}<\mathrm{Br}<\mathrm{NO}_{2}\right)$, as already probed for other MOFs, iii) the location/orientation of the functional group within the framework (i.e. towards the small or large cage), which could modify the accessibility of the metal sites and then, their reactivity and iv) the possible differences in the density of framework defects. [21]

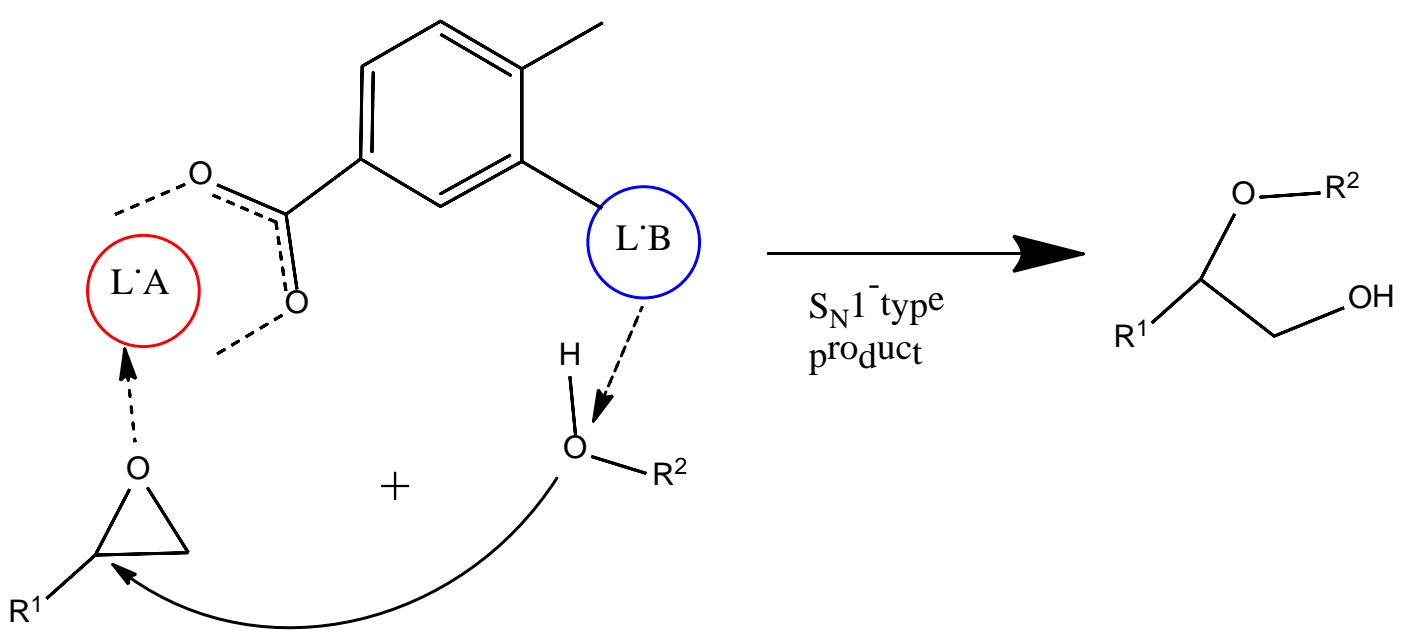

Scheme 1. Dual activation of epoxide ring opening. L.A.: Lewis acid at metal node site, L.B: Lewis basic at the linker substituent. caption is positioned left justified below the figure or scheme. 


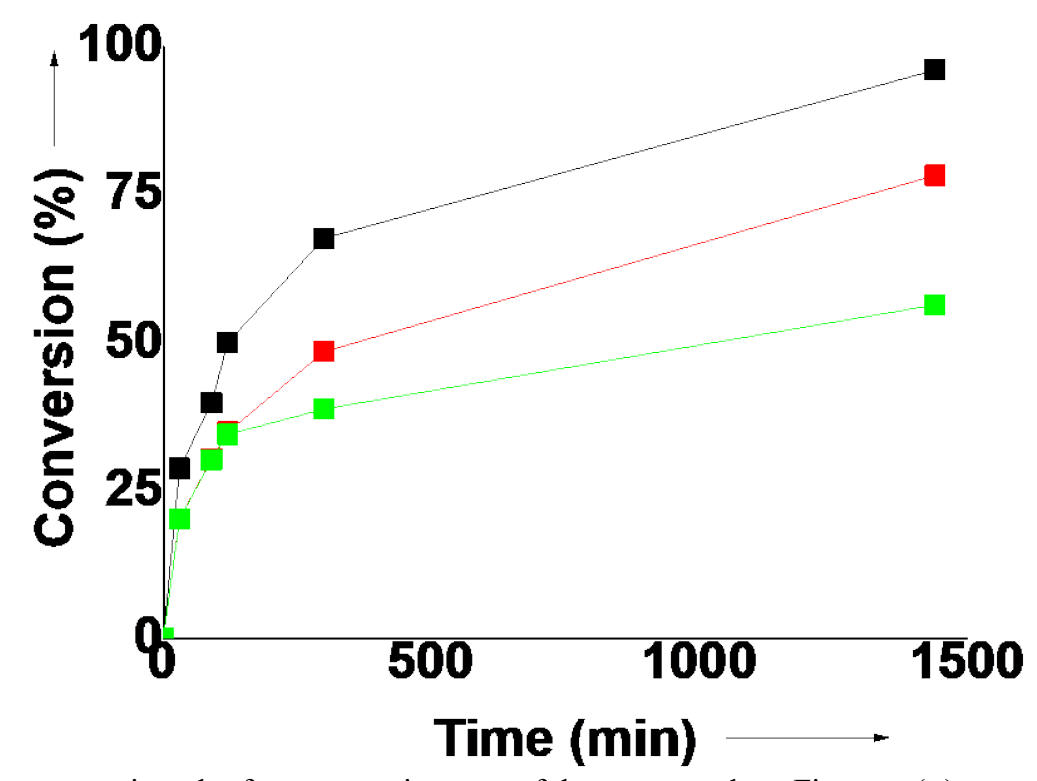

Figure 3. Time-conversion plot for consecutive uses of the same catalyst. First use (घ), second use ( $\square$ ), third use ( $\square$ ). Reaction conditions: styrene oxide (13.14 mmol), methanol (65.7 mL), internal standard (6.57 $\mathrm{mmol}$ ) and catalyst ( $0.486 \mathrm{mmol}$ of metal) caption is positioned left justified below the figure or scheme.

All these parameters, among others, might play a crucial role on the catalytic activity of UiO-66-X, making impossible to find a simple correlation between electronic density at the $\mathrm{Zr}$ ions and catalytic activity. Particularly in the cases of UiO-66 it has been found that depending on the preparation protocol, samples with different crystallinity and defects density can be obtained. There is an increasing interest in determining how the catalytic activity of MOFs depends on lattice defects, particularly in the case of UiO-66. With regard to the dual activation proposed in Scheme 1 there are precedents in the literature suggesting this possibility for instance in the case of the Knovenagel condensation of malonitrile and benzaldehyde in $\mathrm{Cu}_{2}(\mathrm{BTC})_{3}$ as catalyst[22].

Considering the higher activity of UiO-66-Br, this solid was selected for further characterization, including stability and reuse tests.

Heterogeneity of the process was confirmed by performing a hot filtration test, in which after 30 min of the general reaction conditions in the presence of the catalyst, the UiO-66-Br catalyst was filtered off at the reaction temperature when the conversion was ca. 28 \% (see Figure S.3 in supporting information). Then, the resulting clear solution in the absence of the solid catalyst was allowed to continue the reaction. After the removal of UiO-66-Br, the reaction suddenly stops, indicating that the active catalytic species are in the solid. Furthermore, the leaching of the $\mathrm{Zr}$ ions from the UiO-66-X solids during the reaction conditions was ruled out using by determining a very low Zr content $(<0.001 \%)$ in the liquid solution by inductively coupled plasma atomic emission spectroscopy (ICP-AES). This is in agreement with the high chemical stability of the UiO-66$\mathrm{Br}$ under the reaction conditions 
The catalyst stability was also checked by performing a series of consecutive runs using the same UiO-66$\mathrm{Br}$ sample. Thus, the reuse of the sample results in a significant deactivation, associated with a decrease in both the initial reaction rate and the conversion at final time. Although one could consider the catalyst degradation as a possible cause of this deactivation, PXRD of the reused catalyst shows that the crystalline structure of the sample is preserved after its use in two consecutive reactions, ruling out this hypothesis. Thus, we speculated that the significant deactivation of the activity could be due to the presence of strongly adsorbed 2-methoxy-2phenylethanol, acting as poison of the active Lewis sites. To support this hypothesis, after carrying out the catalytic styrene oxide ring opening by methanol, the UiO-66-Br sample was submitted to exhaustive Soxhlet solid-liquid extraction with methanol (see experimental section) before proceeding to a subsequent reuse. The amount of material extracted accounted for presence of the initial styrene oxide weight. Analysis by GC-FID and gas chromatography-mass spectrometry (GC-MS) of the compounds present in the extracted liquor shows the exclusive presence of 2-methoxy-2-phenylethanol, indicating that the UiO-66-Br solid retains some product after the reaction. If phenyl acetaldehyde or other regioisomer of the epoxide ring opening were formed, they should have been detected by our extraction procedure. It is noteworthy that the Soxhlet extraction process renders the solid significantly more active for epoxide ring opening than the analogous sample not submitted to this purification treatment. Despite this extraction, the process was not as efficient to fully recover the initial catalytic activity of the fresh material. After the use and solid-liquid extraction, the time-conversion plot of a subsequent reaction (Figure 3) shows still a gradual decrease in the catalytic activity.

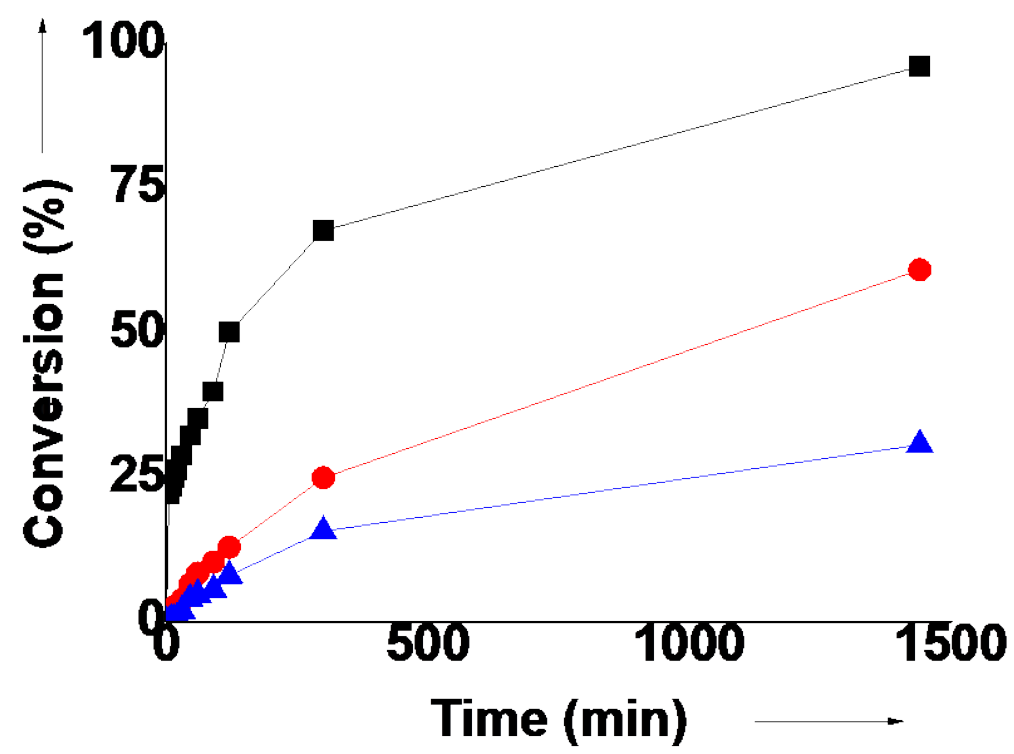

Figure 4. Activity test of fresh UiO-66-Br samples carrying out the reaction in absence ( $\mathbf{\square})$ or presence of $0.30(\bullet)$ or 0.60 ( $\Delta$ ) mmols of 2-methoxy-2-phenylethanol. Reaction conditions: styrene oxide (1 mmol), methanol $(5 \mathrm{~mL})$, internal standard $(0.5 \mathrm{mmol})$ and catalyst $(0.037 \mathrm{mmol}$ of metal).

To provide further confirmation to this hypothesis of deactivation due to catalyst poisoning, a series of three successive catalytic tests were carried out in which the reaction of styrene oxide and methanol was carried out in absence or presence of two different concentrations of 2-methoxy-2-phenylethanol. The results are represented in Figure 4. In presence of 2-methoxy-2-phenylethanol, the reaction rate significantly decreases. The reaction product acts thus as a potent poison on the reaction, substantially decreasing the initial reaction rate of the fresh sample when this compound is present at initial reaction time.

The activation energy for the reaction of styrene oxide and methanol was determined by performing four reactions under the same conditions at different temperatures in the range from 30 to $60{ }^{\circ} \mathrm{C}$. From the temporal evolution of the conversion, the activation energy from the Arrhenius plot was estimated to $27.6 \mathrm{~kJ} \mathrm{~mol}^{-1}$ (Figure 5).

Using $\mathrm{UiO}-66-\mathrm{Br}$ as solid catalyst, the nucleophilic addition to styrene oxide was also screened for a series of primary, secondary and tertiary alcohols, as well as an aromatic and an aliphatic amine. These results are presented in Table 4. Except for the aniline, in all cases a single product corresponding to the regioisomer derived from $S_{N} 1$ mechanism was observed, in agreement with previously shown results. For the case of the aniline, the presence of the regioisomer derived from the attack of the nucleophile to the less substituted carbon 
atom of the epoxide ring was also detected, although with lower selectivity than the $\mathrm{S}_{\mathrm{N}} 1$ product. This is in agreement with the effect of the steric hindrance on the catalytic activity, either of the nucleophile or the functional ligand, as previously proposed. Also using methanol as nucleophile, the reaction of a series of five different asymmetric and symmetric epoxides was investigated. As can be seen in Table 4, the styrene oxide reacts with primary alcohols and the reactivity decreases with the increasing of the chain length (i.e. ethanol, 1propanol and 1-butanol). Iso-propanol also reacts with styrene oxide, but higher reaction temperatures are required to successfully form the product, while for tert-butanol the conversion at much longer reaction time was still significantly lower.
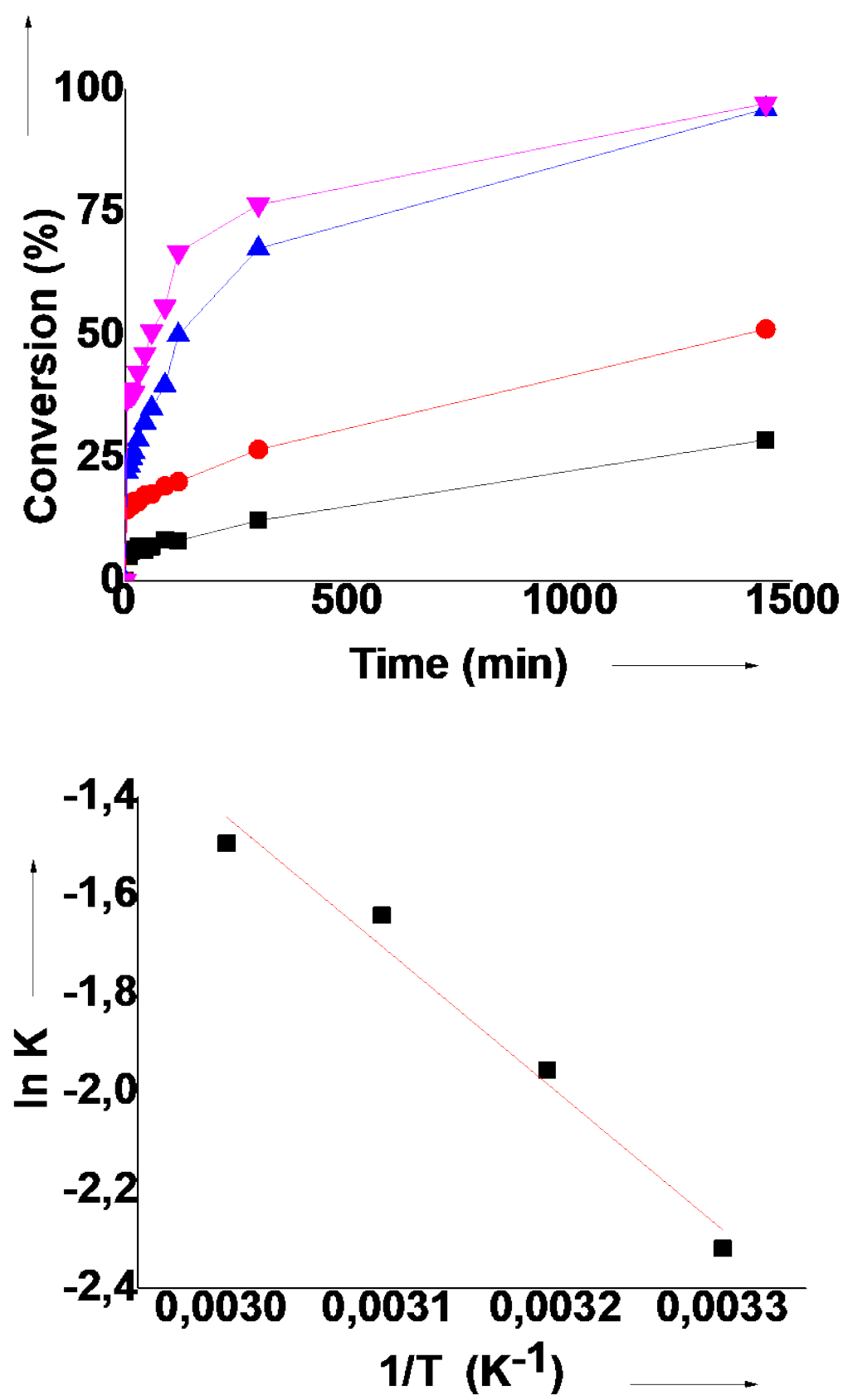

Figure 5. a) Time-conversion plots of epoxide ring opening by UiO-66- $\mathrm{Br}$ at different temperatures (from 30 to $60^{\circ} \mathrm{C}$ ). b) Arrhenius plot of the logarithmic of the initial reaction rate vs the inverse of the absolute temperature. Reaction conditions: styrene oxide $(1 \mathrm{mmol})$, methanol $(5 \mathrm{~mL})$, internal standard $(0.5 \mathrm{mmol})$ and catalyst (0.037 mmol of metal). 
Table 4. Scope of epoxide ring opening for different reagent using UiO66- $\mathrm{Br}$ as catalyst.

\begin{tabular}{|c|c|c|c|c|c|c|}
\hline & Epoxide & Nucleophile & Product & $\mathrm{T}\left({ }^{\circ} \mathrm{C}\right)$ & Conv. (\%) & Yield (\%) \\
\hline 1 & Styrene oxide & Methanol & 2-Methoxy-2-phenylethanol & 50 & 96 & 86 \\
\hline $2^{b}$ & Styrene oxide & Ethanol & 2-Ethoxy-2-phenylethanol & 50 & 44 & 41 \\
\hline $3^{\mathrm{b}}$ & Styrene oxide & Etanol & 2-Ethoxy-2-phenylethanol & Reflux & 65 & 58 \\
\hline 4 & Styrene oxide & Propanol & 2-Phenyl-2-propoxylethanol & 50 & 43 & 40 \\
\hline $5^{\mathrm{b}}$ & Styrene oxide & Propanol & 2-Phenyl-2-propoxylethanol & Reflux & 91 & 87 \\
\hline 6 & Styrene oxide & n-Butanol & 2-Butoxy-2-phenylethanol & 50 & 12 & 10 \\
\hline 7 & Styrene oxide & $n$-Butanol & 2-Butoxy-2-phenylethanol & Reflux & 91 & 88 \\
\hline 8 & Styrene oxide & i-Propanol & 2-isopropoxy-2-phenylethanol & 50 & -- & -- \\
\hline 9 & Styrene oxide & i-Propanol & 2-isopropoxy-2-phenylethanol & Reflux & 97 & 96 \\
\hline $10^{\mathrm{b}}$ & Styrene oxide & $t$-Butanol & $\begin{array}{l}\text { 2-(tert-butoxy)-2- } \\
\text { phenylethanol }\end{array}$ & 50 & 8 & 4 \\
\hline $11^{\mathrm{b}}$ & Styrene oxide & $t$-Butanol & $\begin{array}{l}\text { 2-(tert-butoxy)-2- } \\
\text { phenylethanol }\end{array}$ & Reflux & 49 & 40 \\
\hline 12 & Styrene oxide & Aniline & $\begin{array}{l}\text { 2-phenyl-2- } \\
\text { (phenylamonio)ethanol }\end{array}$ & Reflux & 54 & 51 \\
\hline 13 & Styrene oxide & $\begin{array}{l}n- \\
\text { Hexylamine }\end{array}$ & $\begin{array}{l}\text { 2-(heyxlamonio)-2- } \\
\text { phenylethanol }\end{array}$ & Reflux & 90 & 90 \\
\hline 14 & $\begin{array}{l}\text { 2,2- } \\
\text { Dimethyloxir } \\
\text { ane }\end{array}$ & Methanol & $\begin{array}{l}\text { 1-Methoxy-2-methylpropan- } \\
\text { 2-ol }\end{array}$ & 50 & 42 & 40 \\
\hline $15^{\mathrm{b}}$ & $\begin{array}{l}\text { Cyclohexene } \\
\text { oxide }\end{array}$ & Methanol & 2-Methoxycyclohexanol & 50 & 50 & 47 \\
\hline 16 & $\begin{array}{l}\text { Cyclopentene } \\
\text { oxide }\end{array}$ & Methanol & 2-Methoxycyclopentanol & 50 & 61 & 46 \\
\hline 17 & Epoxyhexane & Methanol & 1-Methoxyhexan-1-ol & 50 & 22 & 20 \\
\hline $18^{\mathrm{b}}$ & $\begin{array}{l}\text { Stillbene } \\
\text { oxide }\end{array}$ & Methanol & $\begin{array}{l}\text { 2-Methoxy-1,2- } \\
\text { diphenylethanol }\end{array}$ & 50 & 13 & 11 \\
\hline
\end{tabular}

a) Reaction conditions: Catalyst (13.4 mg), epoxide (1 mmol), alcohol (2.5 mL), internal standard (0.5 $\mathrm{mmol}), 24 \mathrm{~h}$. Conversion were calculated by GC using internal standard and yield were calculated by ${ }^{1} \mathrm{H}-$ NMR spectroscopy. b) Time reaction $48 \mathrm{~h}$.

All these reactivity patterns are consistent with the negative influence of steric hindrance of the alcohol on the final reactivity. Additionally, aliphatic hexylamine reacts to give the corresponding $\alpha, \beta$-aminoalcohol faster than the aromatic amine, in agreement with the relative nucleophilicity of the amine. 
Finally, regarding the structure of the epoxide, styrene oxide having a benzylic position reacts much faster than aliphatic monosubstituted or disubstituted aliphatic epoxides. Further, stilbene oxide is the epoxide of the series that reacts the slowest due to a combination of steric encumbrance and electronic effects.

\section{Conclusion}

In the present study, it has been shown that the catalytic activity of the microporous zirconium terephthalate UiO-66 can be tuned by introducing functional groups on the aromatic ring of the terephthalate linker; the initial reaction rate varying by more than two orders of magnitude depending of the substituent. A linear correlation between the values of the Hammett constant of the substituent and the initial reaction rate does not however apply in the present case, suggesting that the reaction mechanism might involve more than one type of center or that other factors besides electron density have to be considered. The most active material was the $\mathrm{UiO}-66-\mathrm{Br}$ derivative that behaves as heterogeneous catalyst and exhibits a scope in agreement with the expected influence of the substrate and nucleophile reactivity. Although the chemical and structural integrity of the catalyst is maintained under the reaction conditions, deactivation of the catalyst is observed, probably due to poisoning by the reaction product. Overall, this study constitutes an additional proof that more efficient MOF catalysts can be simply obtained by changing the chemical nature of isostructural MOFs thorough ligand functionalization.

\section{Acknowledgments}

Financial support by the Spanish Ministry of Economy and Competitiveness (Severo Ochoa and CTQ201569153) and Generalitat Valenciana (Prometeo 2013/14) is grateful acknowledged. We also thank EU under the Being Energy contract for partial funding. J.F.B. thanks the Universidad Politécnica de Valencia for a postgraduate scholarship. S.N. thanks the Spanish Ministerio de Educación, Cultura y Deporte for José Castillejo mobility programme (CAS14/00067). 


\section{References}

[1] (a) T. Devic, C. Serre, Chem.Soc.Rev. High valence 3p and transition metal based MOFs. 43, (2014), 6097-6115; (b) G. Ferey, C. Mellot-Draznieks, C. Serre, F. Millange, J. Dutour, S. Surble, I. Margiolaki, Science, A chromium terephthalate-based solid with unusually large pore volumes and surface area. 309, (2005), 2040-2042; (c) S. Kitagawa, R. Kitaura, S.-I. Noro, Angew. Chem., Int. Ed. Functional porous coordination polymers. 43, (2004), 2334-2237.

[2] H. Furukawa, Cordova, K.E., O'Keeffe, M., Yaghi, O.M., Science. The Chemistry and Applications of Metal-Organic Frameworks. 341, (2013), 1230444.

[3] (a)A. Corma, H. Garcia, F. X. Llabrés i Xamena, Chem. Rev. Engineering Metal Organic Frameworks for Heterogeneous Catalysis. 110, (2010), 4606-4655; (b) M. Yoon, R. Srirambalaji, K. Kim, Chem. Rev. Homochiral Metal-Organic Frameworks for Asymmetric Heterogeneous Catalysis. 112, (2012), 1196-1231.

[4] J. Gascon, A. Corma, F. Kapteijn, F. X. Llabrés i Xamena, ACS Catal. Metal Organic Framework Catalysis: Quo vadis?. 4, (2014), 361-378.

[5] (a) D. Farrusseng, S. Aguado, C. Pinel, Angew. Chem. Int. Ed. Metal-Organic Frameworks: Opportunities for Catalysis. 41, (2009), 7502-7513; (b) A. Dhakshinamoorthy, M. Alvaro, H. Garcia, Chem. Commun. Commercial metal-organic frameworks as heterogeneous catalysts. 48, (2012), 11275-11288; (c) A. Dhakshinamoorthy, H. Garcia, Chem. Soc. Rev. Catalysis by metal nanoparticles embedded on metal-organic frameworks. 41, (2012), 5262-5284; (d) M. Ranocchiari, Bokhoven, J.A.V., Phys. Chem. Chem. Phys. Catalysis by metal-organic frameworks: fundamentals and opportunities. 13, (2011), 6388-6396.

[6] A. Dhakshinamoorthy, Opanasenko, M., Čejka, J., Garcia, H., Catal. Sci. Technol. Metal organic frameworks as heterogeneous catalysts for the production of fine chemicals. 3, (2013), 2509-2540.

[7] J. Liu, L. Chen, H. Cui, J. Zhang, L. Zhang, C.-Y. Su, Chem. Soc. Rev. Applications of metal-organic frameworks in heterogeneous supramolecular catalysis. 43, (2014), 6011-6061.

[8] O. A. Kholdeeva, I. Y. Skobelev, I. D. Ivanchikova, K. A. Kovalenko, V. P. Fedin, A. B. Sorokin, Catal. Today Hydrocarbon oxidation over Fe-and Cr-containing metal-organic frameworks MIL-100 and MIL-101-a comparative study. 238, (2014), 54-61.

[9] A. Dhakshinamoorthy, M. Alvaro, P. Horcajada, E. Gibson, M. Vishnuvarthan, A. Vimont, J.-M. Grenèche, C. Serre, M. Daturi, H. Garcia, ACS Catal. Comparison of Porous Iron Trimesates Basolite F300 and MIL100(Fe) As Heterogeneous Catalysts for Lewis Acid and Oxidation Reactions: Roles of Structural Defects and Stability. 2, (2012), 2060-2065.

[10] (a)I. Y. Skobelev, A. B. Sorokin, K. A. Kovalenko, V. P. Fedin, O. A. Kholdeeva, J. Catal. Solvent-free allylic oxidation of alkenes with O 2 mediated by Fe-and Cr-MIL-101. 298, (2013), 61-69; (b) N. V.

Maksimchuk, K. A. Kovalenko, V. P. Fedin, O. A. Kholdeeva, Chem. Comm. Cyclohexane selective oxidation over metal-organic frameworks of MIL-101 family: superior catalytic activity and selectivity. 48, (2012), 6812-6814; (c) I. Y. Skobelev, K. A. Kovalenko, V. P. Fedin, A. B. Sorokin, O. A. Kholdeeva, Kinet. Catal. Allylic oxidation of alkenes with molecular oxygen catalyzed by porous coordination polymers Fe-MIL-101 and Cr-MIL-101. 54, (2013), 607-614; (d) A. Gómez-Paricio, A. Santiago-Portillo, S. Navalón, P. Concepción, M. Alvaro, H. Garcia, Green Chem. MIL-101 promotes the efficient aerobic oxidative desulfurization of dibenzothiophenes. 18 (2016), 508-515; (e) Z. Saedi, S. Tangestaninejad, M. Moghadam, V. Mirkhani, I. Mohammadpoor-Baltork, Catal. Comm. MIL-101 metal-organic framework: A highly efficient heterogeneous catalyst for oxidative cleavage of alkenes with H2O2. 17, (2012), 18-22; (f) A. Santiago-Portillo, S. Navalon, F. Cirujano, F. Llabrés i Xamena, M. Alvaro, H. Garcia, ACS Catal. MIL-101 as reusable solid catalyst for autooxidation of benzylic hydrocarbons in the absence of additional oxidizing reagent. 5, (2015), 3216-3224.

[11] (a) U. S. F. Arrozi, H. W. Wijaya, A. Patah, Y. Permana, Appl. Catal. A Gen. Efficient acetalization of benzaldehydes using UiO-66 and UiO-67:Substrates accessibility or Lewis acidity of zirconium. 506, (2015), 77-84; (b) J. Chen, K. Li, L. Chen, R. Liu, X. Huang, D. Ye, Green Chem. Conversion of fructose into 5hydroxymethylfurfural catalyzed by recyclable sulfonic acid-functionalized metal-organic frameworks. 16, (2014), 2490-2499; (c) F. G. Cirujano, A. Corma, F. X. Llabrés i Xamena, Chem. Eng. Sci. Conversion of Levulinic acid into chemicals: Synthesis of biomass derived levulinate esters over Zr-containing MOFs. 124, (2015), 52-60; (d) J. Hajek, M. Vandichel, B. Van De Voorde, B. Bueken, D. De Vos, M. Waroquier, V. Van Speybroeck, J. Catal. Mechanistic studies of aldol condensations in UiO-66 and UiO-66-NH2 metal organic frameworks. 331, (2015), 1-12; (e) Y. Luan, N. Zheng, Y. Qi, J. Yu, G. Wang, Eur. J. Inorg. Chem. Development of a SO3H-Functionalized UiO-66 Metal-Organic Framework by Postsynthetic Modification and Studies of Its Catalytic Activities 26, (2014), 4268-4272; (f) V. N. Panchenko, M. M. Matrosova, J. Jeon, J. W. Jun, M. N. Timofeeva, S. H. Jhung, J. Catal. Catalytic behavior of metal-organic frameworks in the Knoevenagelcondensation reaction. 316, (2014), 251-259; (g) F. Vermoortele, B. Bueken, G. Le Bars, B. Van De Voorde, M. Vandichel, K. Houthoofd, A. Vimont, M. Daturi, M. Waroquier, V. Van Speybroeck, C. Kirschhock, D. E. De Vos, J. Am. Chem. Soc. Synthesis modulation as a tool to increase the catalytic activity 
of metal-organic frameworks: the unique case of UiO-66(Zr).135, (2013), 11465-11468; (h) F. G. Xi, Y. Yang, H. Liu, H. F. Yao, E. Q. Gao, RSC Adv. Different acidity and additive effects of zirconium metal-organic frameworks as catalysts for cyanosilylation. 5, (2015), 79216-79223; (i) Y. Yang, H. F. Yao, F. G. Xi, E. Q. Gao, J. Mol. Catal. A Chem. Amino-functionalized Zr(IV) metal-organic framework as bifunctional acid-base catalyst for Knoevenagel condensation. 390, (2014), 198-205; (j) J. Ye, J. K. Johnson, ACS Catal. Screening Lewis Pair Moieties for Catalytic Hydrogenation of CO2 in Functionalized UiO-66. 5, (2015), 6219-6229.

[12] F. Vermoortele, R. Ameloot, A. Vimont, C. Serre, D. De Vos, Chem. Commun. An amino-modified Zrterephthalate metal-organic framework as an acid-base catalyst for cross-aldol condensation. 47, (2011), 15211523.

[13] (a) M. Lammert, S. Bernt, F. Vermoortele, D. E. De Vos, N. Stock, Inorg. Chem. Single-and mixed-linker Cr-MIL-101 derivatives: A high-throughput investigation. 52, (2013), 8521-8528; (b) N. Stock, S. Biswas, Chem. Rev. Synthesis of Metal-Organic Frameworks (MOFs): Routes to Various MOF Topologies, Morphologies, and Composites. 112, (2012), 933-969; (c) C. Janiak, J. K. Vieth, New J. Chem. MOFs, MILs and more: concepts, properties and applications for porous coordination networks (PCNs). 34, (2010), 23662388.

[14] J. G. Vitillo, E. Groppo, S. Bordiga, S. Chavan, G. Ricchiardi, A. Zecchina, Inorg. Chem. tability and Reactivity of Grafted Cr(CO)3 Species on MOF Linkers: A Computational Study. 48, (2009), 5439-5448.

[15] F. Vermoortele, M. Vandichel, B. V. de Voorde, R. Ameloot, M. Waroquier, V. Van Speybroeck, D. E. De Vos, Angew. Chem. Int. Ed. Electronic Effects of Linker Substitution on Lewis Acid Catalysis with MetalOrganic Frameworks. 51, (2012), 4887-4890.

[16] (a) Cavka, J. H.; Jakobsen, S.; Olsbye, U.; Guillou, N.; Lamberti, C.; Bordiga, S.; Lillerud, K. P. J. Am. Chem. Soc. A new zirconium inorganic building brick forming metal organic frameworks with exceptional stability. 130, (2008), 13850-13851. (b) J. Canivet, M. Vandichel and D. Farrusseng, Dalton Trans. Origin of highly active metal-organic framework catalysts: defects? Defects!. 45, (2016), 4090-4099. (c) Morris R. E., Čejka, J. Natture Chem. Exploiting chemically selective weakness in solids as a route to new porous materials. 7, (2015), 381-388

[17] A. Dhakshinamoorthy, M. Alvaro, H. Garcia, Catal. Sci. Technol. Metal-organic frameworks as heterogeneous catalysts for oxidation reactions. 1, (2011), 856-867.

[18] (a) Shen, L. Liang, R. Luo, M. Jing, F. Wu, L. PCCP. Electronic effects of ligand substitution on metalorganic framework photocatalysts: the case study of UiO-66. 17, (2015), 117-121. (b) Kandiah, M., Nilsen, M.H., Usseglio, S., Jakobsen, S., Olsbye, U., Tilset, M., Larabi, C., Quadrelli, E.A., Bonino, F., Lillerud, K.P. Chem. Mater. Synthesis and Stability of Tagged UiO-66 Zr-MOFs. 24, (2010), 6632-6640.

[19] D. Cunha, C. Gaudin, I. Colinet, P. Horcajada, G. Maurin, C. Serre, J. Mater. Chem. B 1, Rationalization of the entrapping of bioactive molecules into a series of functionalized porous zirconium terephthalate MOFs . 1, (2013), 1101-1108.

[20] A. Dhakshinamoorthy, M. Alvaro, P. Concepción, V. Fornés, H. Garcia, Chem. Commun. Graphene oxide as an acid catalyst for the room temperature ring opening of epoxides. 48, (2012), 5443-5445.

[21] (a) Charton, M. Top. Curr. Chem. The upsilon steric parameter - definition and determination 114, (1983), 57. (b) Devic, T.; Horcajada, P.; Serre, C.; Salles, F.; Maurin, G.;Moulin, B.; Heurtaux, D.; Clet, G.; Vimont, A.; Greneche, J.-M.; Le Ouay, B.; Moreau, F.; Magnier, E.; Filinchuk, Y.; Marrot, J.; Lavalley, J.-C.; Daturi, M.; Ferey, G. J. Am. Chem. Soc. Functionalization in flexible porous solids: effects on the pore opening and the host-guest interactions. 132, (2010), 1127-36.

[22] M. Položij, M. Rubeš, J. Čejka, P. Nachtigall. ChemCatChem, Catalysis by Dynamically Formed Defects in a Metal-Organic Framework Structure: Knoevenagel Reaction Catalyzed by Copper Benzene-1, 3, 5tricarboxylate. 10, (2014), 2821-2824

\section{Supplementary Material}




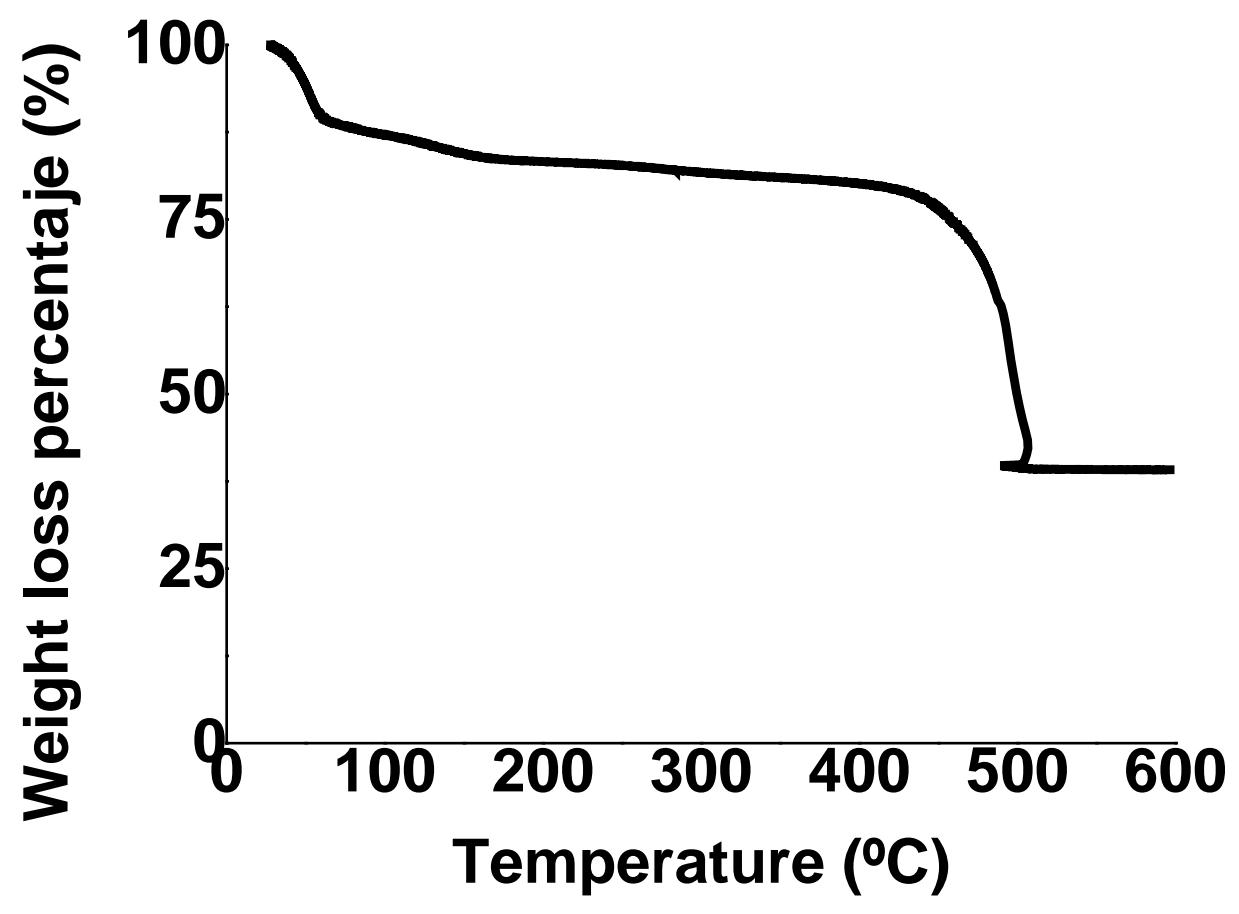

$\underline{\mathrm{UiO}-66-\mathrm{NO}_{2}}$

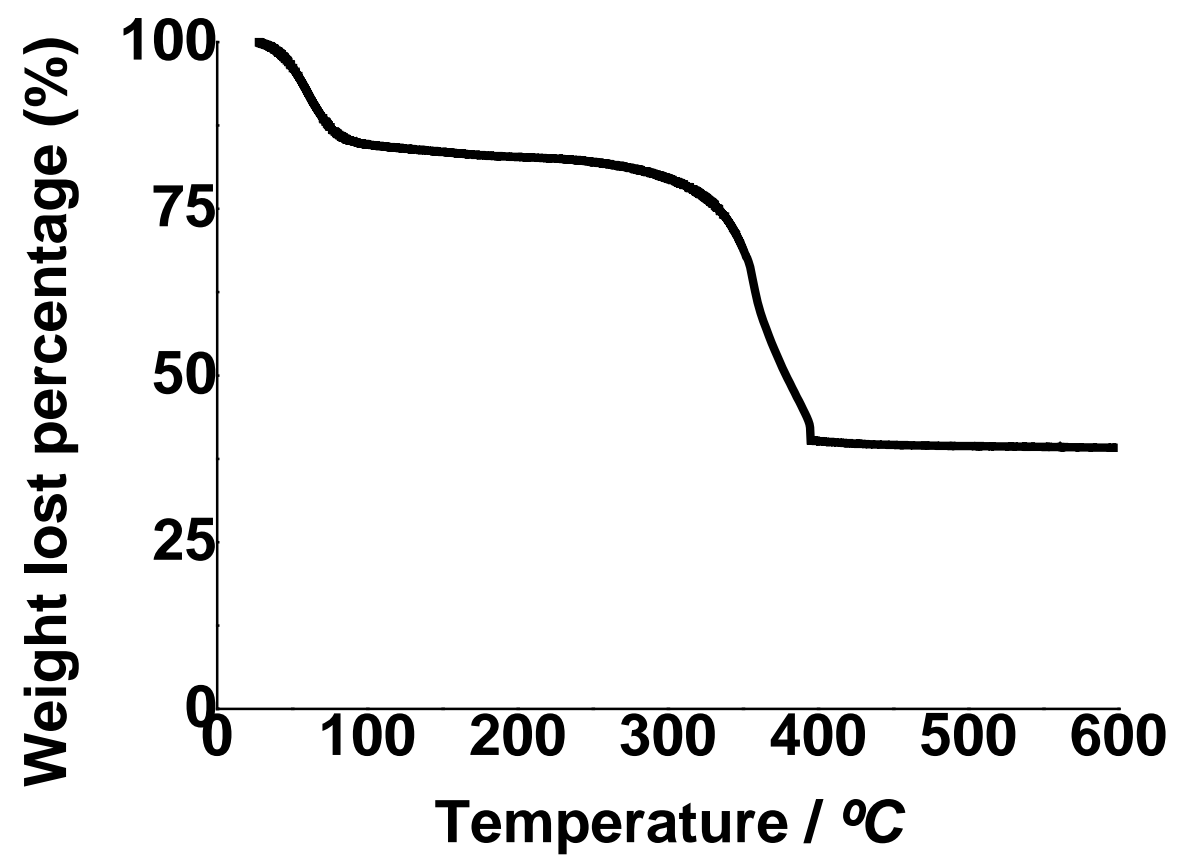

$\underline{\text { UiO-66-NH2 }}$ 


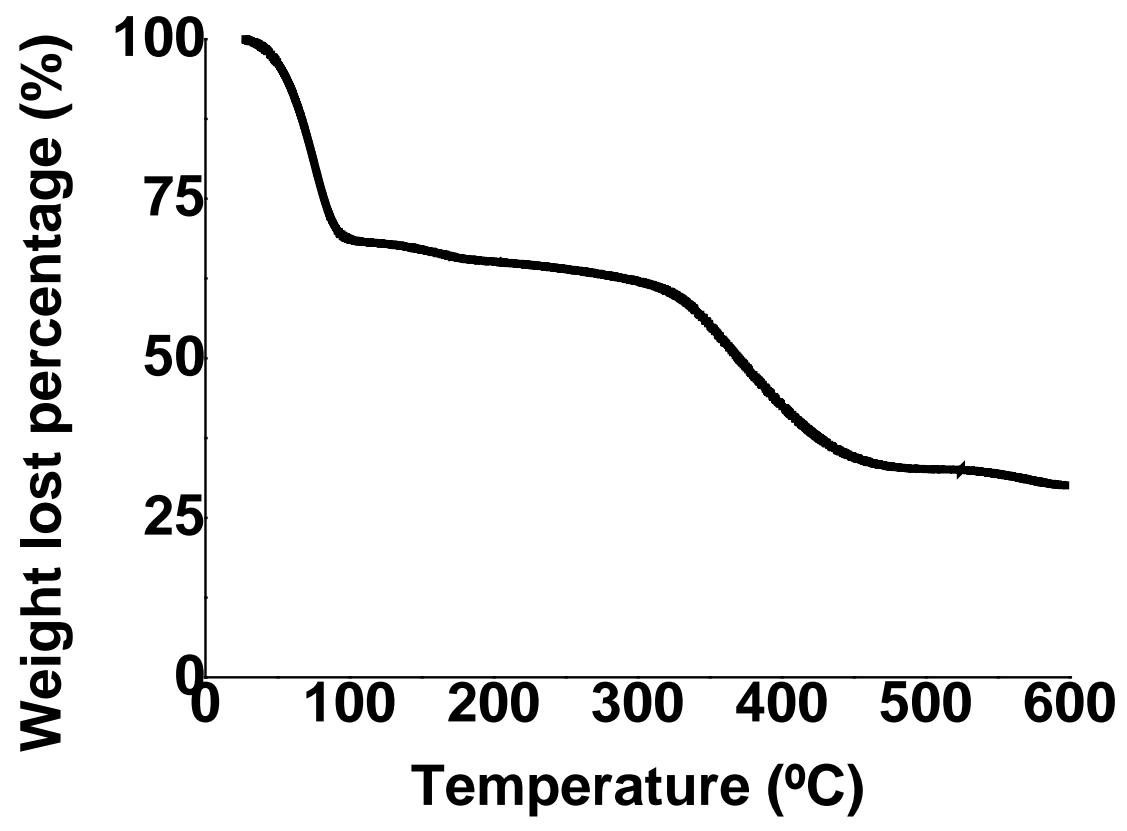

$\underline{\text { UiO-66-Br }}$

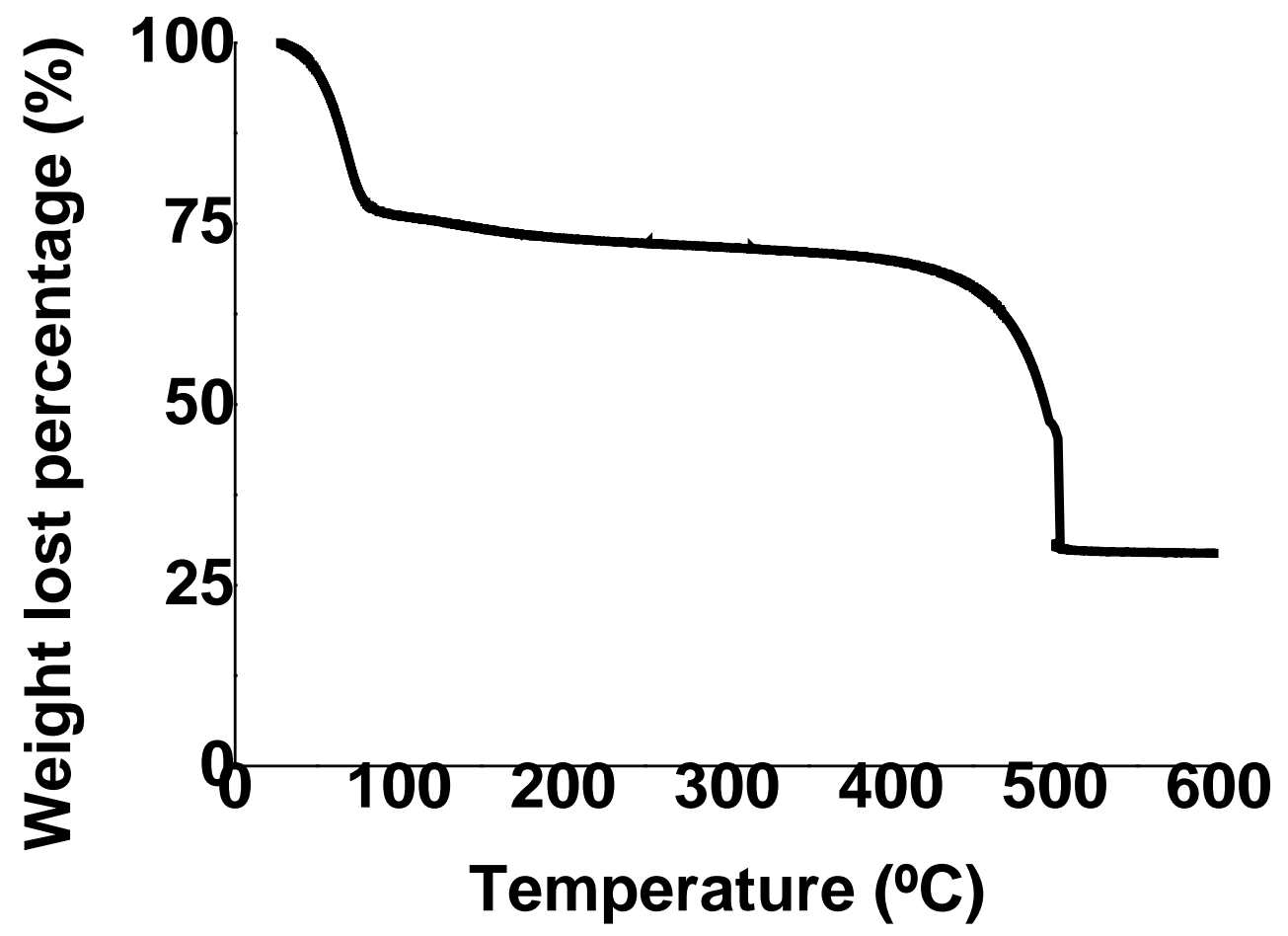




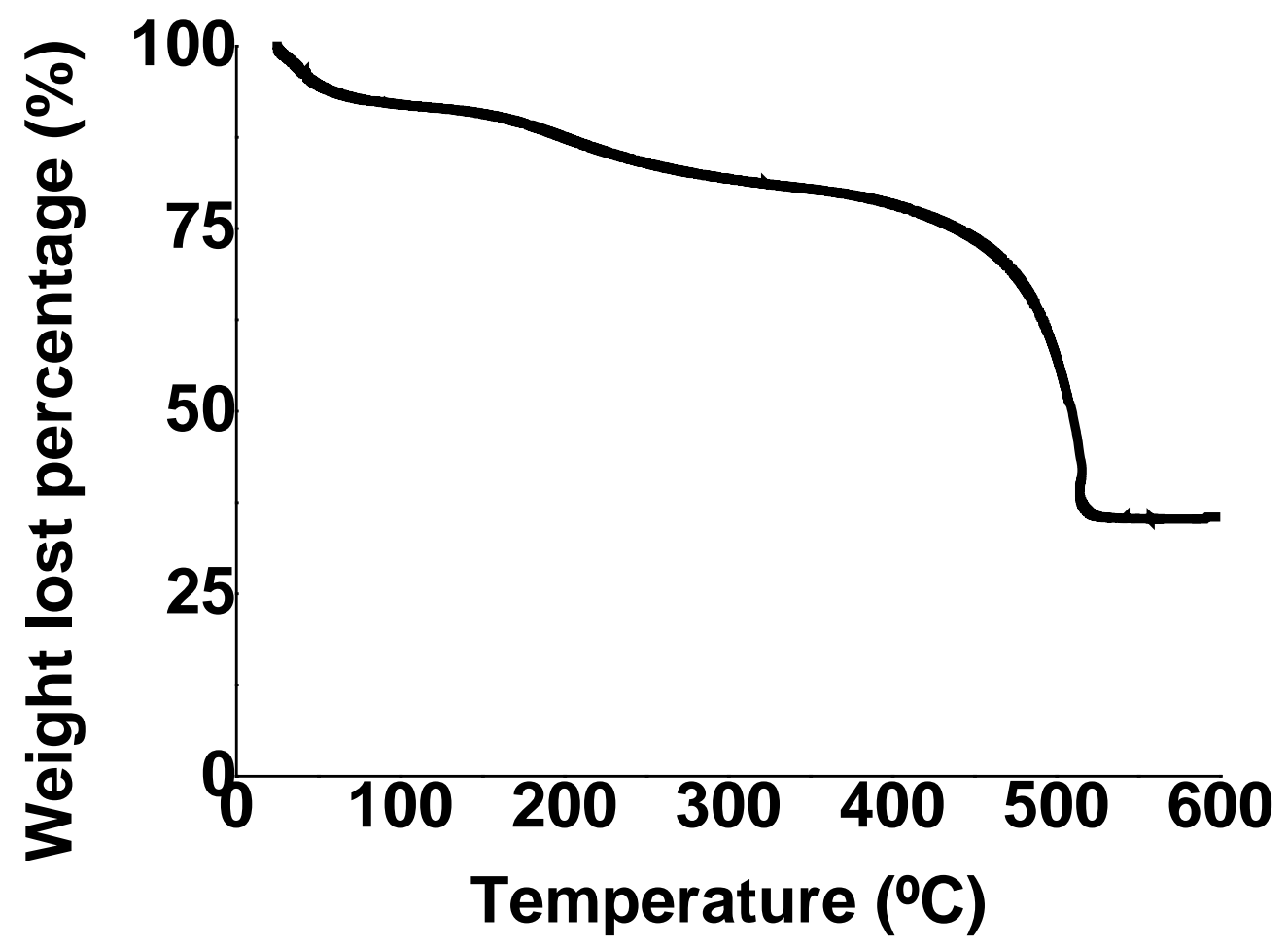

Figure S.1. Thermogravimetric profile of the series of UiO-66-X under study
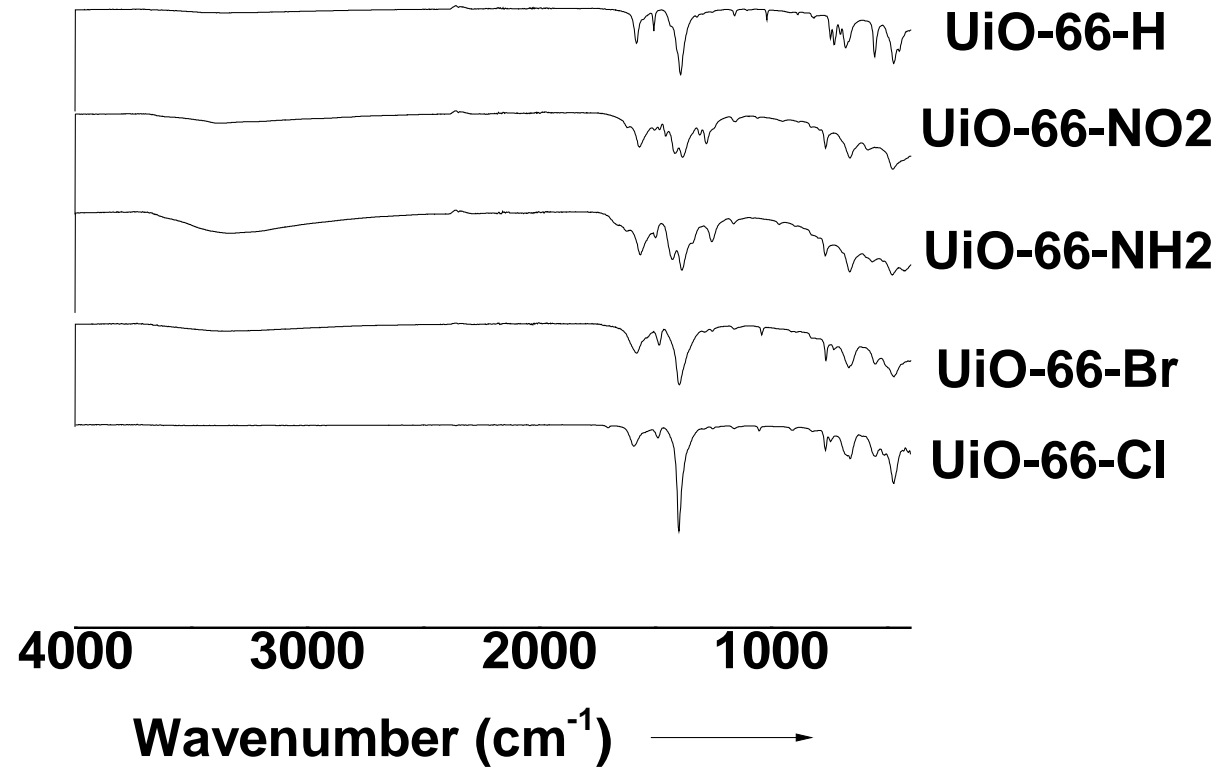

Figure S.2. IR spectra for the series of UiO-66-X. From top to the bottom: UiO-66-H, UiO-66- $\mathrm{NO}_{2}, \mathrm{UiO}-66-\mathrm{NH}_{2}$, UiO66-Br, UiO-66-Cl. 


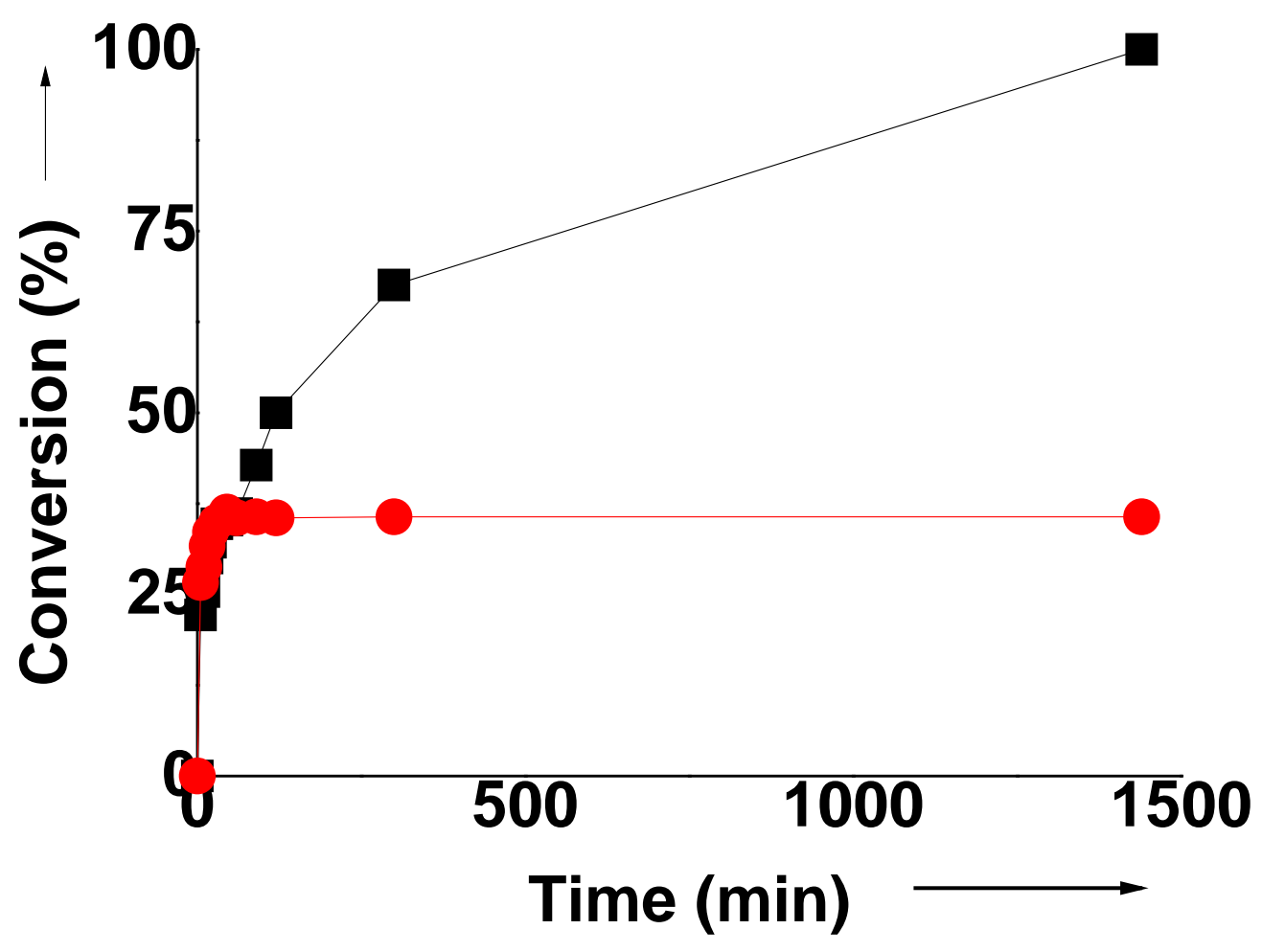

Figure S. 3. Hot filtration test. Reaction conditions: styrene oxide (1 mmol), methanol (5 mL), internal standard $(0.5 \mathrm{mmol})$ and catalyst $(0.037 \mathrm{mmol}$ of metal). Reactions carried out in normal conditions ( $\mathbf{(})$ and reaction carried out in normal condition and catalyst was removed after $30 \mathrm{~min}(\bullet)$. 